reported from the Bath Unit for Research into Paediatrics, Royal United Hospital, Combe Park, Bath, Avon, England and Bristol MRI Centre, Frenchay Hospital, Bristol. Nine patients had between 2 and 9 cerebral tubers seen on MRI T2 weighted images, 7 had 1-5 subependymal nodules and 2 had normal scans. The hamartomas were at least $4 \mathrm{~mm}$ in size. The number of lesions found on the MRI may not be used to predict neurological outcome in tuberous sclerosis. Only 3 of the 11 patients had a history of seizures and all had normal intellect (Webb DW et al. Cranial magnetic resonance imaging in patients with tuberous sclerosis and normal intellect. Arch Dis Child; Dec 1991; 66:1375-1377). (Reprints: Dr. Osborne, Royal United Hospital, Comb Park, Bath, Avon BA1 3NG, England.)

COMMENT. The authors suggest that the wrong conclusions may be drawn if the number of lesions alone on cranial MRI is used to predict neurological outcome in tuberous sclerosis. Lesions seen on both MRI and CT may become less obvious with advancing age in patients with tuberous sclerosis. In the experience of these authors, individuals who reach 5 years of age with normal development do not develop mental handicap from tuberous sclerosis. While the number of cerebral hamartomas appears to be an unreliable predictor of clinical outcome it is possible that the distribution and size of lesions may be more helpful. Patients with temporal lobe or occipital lobe hamartomas are most likely to have seizures or EEG abnormalities. Tuberous sclerosis was the cause of infantile spasms in $10 \%$ of cases treated in Finland (see Ped Neurol Briefs April 1990) but in only 2\% reported from China (Feng YK et al. Chinese Med J. May 1991; 104:416-421).

\title{
ANTIEPILEPTIC DRUGS
}

\section{VALPROATE FOR INTRACTABLE EPILEPSY}

The efficacy and side effects of high dose valproic acid (VPA) therapy in 46 children with refractory epilepsy were studied at the Department of Child Neurology, Okayama University Medical School, Okayama, Japan. Serum VPA concentrations ranged from 105.1 to $198.4 \mathrm{mcg} / \mathrm{ml}$. Seizures were completely controlled in $15(32.6 \%)$ and improved in $12(26.1 \%)$. This degree of control was sustained at 6 months or more follow-up. VPA therapy was especially effective for West syndrome and for epilepsy with continuous spike waves during slow wave sleep. Symptomatic generalized epilepsy and symptomatic partial epilepsy were benefitted equally. No significant difference in efficacy was noted between the monotherapy and bipharmacy groups, but adverse side effects appeared more frequently and higher serum levels of VPA were difficult to obtain in the bipharmacy group. Liver enzymes were transiently increased in $50 \%$ of the patients and elevated serum amylase occurred in $10 \%$ of patients. Hyperammonemia in $6.5 \%$ of patients necessitated withdrawal of the drug. Thrombocytopenia less than $100,000 / \mathrm{mm}^{3}$ occurred in $21.7 \%$ and hypofibrinogenemia was detected in $65 \%$ of patients. These side effects were reversible with reduction of dosage 
(Ohtsuka Y, Ohtahara S et al. Treatment of intractable childhood epilepsy with high-dose valproate. Epilepsia Jan/Feb 1992; $\underline{33}$ :158-164). (Reprints: Dr. Y. Ohtsuka, Department of Child Neurology, Okayama University Medical School, 2-5-1, Shikata-cho, Okayama 700 , Japan.)

COMMENT. The authors recommend high dose VPA therapy for intractable childhood epilepsy of generalized and partial types and particularly for patients with West syndrome. The monitoring of platelet counts and fibrinogen levels in addition to liver function tests is advised.

Kreuz W et al. from Frankfort, Germany report the induction of von Willebrand disease type $I$ in $67 \%$ of 30 children receiving VPA therapy (Epilepsia Jan/Feb 1991; 33:178-184). The decrease in coagulation parameters were not dependent on either VPA dose or the period of drug administration. This increased tendency to hemorrhage in patients receiving VPA must be considered, especially during surgical intervention and after traumatic events.

Kondo $T$ et al. from Hirosaki University, Japan, report that 3 risk factors (young age, polypharmacy, and high VPA serum level) enhance the susceptibility to VPA hepatotoxicity by altering the metabolism of VPA and by increasing the conversion of VPA to 4-en, the most toxic VPA metabolite (Epilepsia Jan/Feb 1992; $\underline{33}$ :172-177).

\section{CARBAMAZEPINE-INDUCED DIARRHEA}

Intractable diarrhea induced by carbamazepine (CBZ) in 3 patients and necessitating discontinuation of the drug is reported from the Departments of Neurology and Medicine, University of Louisville School of Medicine, Kentucky. A 3 year old boy with generalized tonic-clonic seizures received CBZ $125 \mathrm{mg} / \mathrm{day}$, increasing to $125 \mathrm{mg} 3$ times daily. The CBZ serum level was $7.4 \mathrm{mcg} / \mathrm{ml}$. Frequent diarrhea was associated with negative stool examination and cultures. Liver function studies showed minimal LDH elevation. The gradual withdrawal of $\mathrm{CBZ}$ and substitution of phenytoin were followed promptly by recovery. The 2 other patients were adults, both of whom recovered after CBZ was withdrawn and phenytoin or primidone was substituted (Iyer V, Holmes JW, Richardson RL. Intractable diarrhea from carbamazepine. Epilepsia Jan/Feb 1992; 33:185-187). (Reprints: Dr. V. Iyer, Department of Neurology, University of Louisville, Louisville, KY 40292.)

COMMENT. The authors note that diarrhea is described as a complication of CBZ in the package insert but gastrointestinal adverse effects of $\mathrm{CBZ}$ have received little attention in the literature. Other GI complications include anorexia, nausea, vomiting and constipation. Hepatic toxicity has been reported in $5 \%-10 \%$ of patients.

Carbamazepine-induced eosinophilic colitis with severe watery diarrhea is reported in an adult from Helsinki, Finland (Anttila VJ, Valtonen M. Epilepsia Jan/Feb 1992; 33:119-121). With 2 reports of 